\title{
Environmental control of morphine tolerance in the hamster
}

\author{
PAUL SCHNUR and RAE ANN MARTINEZ \\ University of Southern Colorado, Pueblo, Colorado
}

\begin{abstract}
Tolerance to morphine-induced hypoactivity in hamsters was investigated under conditions designed to test a Pavlovian conditioning model of morphine tolerance. One group of animals received i.p. injections of morphine $(50 \mathrm{mg} / \mathrm{kg})$ in the test environment and saline in the home cage; a second group received saline in the test environment and morphine in the home cage; a third group received saline in both environments. A subsequent morphine challenge in the test environment gave evidence of both associative and nonassociative tolerance. Associative tolerance was detectable 1 week later during a second morphine challenge. Compensatory hyperactivity, however, was not observed during a saline challenge in the presence of morphine-associated cues. Following the acquisition of tolerance, nonreinforced exposure to morphine-associated cues produced an attenuation of morphine tolerance (i.e., extinction of tolerance). The results are interpreted as providing partial support for the Pavlovian model and are discussed in terms of alternative associative models of tolerance.
\end{abstract}

Considerable evidence now exists that learning processes modulate the development or expression of tolerance to the analgesic, pyretic, and locomotor effects of morphine (Baker \& Tiffany, 1985; Goudie \& Demellweek, 1986; Siegel, 1979, 1983; Stewart \& Eikelboom, 1987). According to a Pavlovian conditioning model of learned tolerance (Siegel, 1983), drug administration constitutes a Pavlovian conditioning trial in which an association between situational cues (the conditioned stimuli, CSs) and morphine (the unconditioned stimulus, UCS) gives rise to a compensatory conditioned response (CR) that counteracts the drug-induced unconditioned response (UCR). Consistent with this view is the reliable finding that morphine tolerance is situation-specific: Animals challenged with a test dose of morphine in the same environment in which they have experienced previous doses of morphine are more tolerant to morphine's effects than animals exposed to the same amount and schedule of morphine but tested in a different environment (Fanselow \& German, 1982; Hinson \& Siegel, 1983; Mucha, Volkovskis, \& Kalant, 1981; Siegel, 1975, 1976, 1977; Tiffany \& Baker, 1981; Tiffany \& Maude-Griffin, 1988). Moreover, environmental manipulations that modify Pavlovian conditioning similarly affect the acquisition of morphine tolerance. For example, morphine tolerance is subject to such Pavlovian conditioning effects as extinction, latent inhibition, partial reinforcement, overshadowing, conditioned inhibition, and blocking (Siegel, 1979, 1983). Finally,

This research was supported by NIH Minority Biomedical Research Support Grant (RR-08197-05) to the University of Southern Colorado (Paul Schnur, principal investigator). Morphine sulfate was generously donated by Mallinckrodt, Inc. (St. Louis, MO). The authors would like to thank David Hang and Charlotte Archuletta for their assistance in data collection. Correspondence should be addressed to Paul Schnur, who is now at the Center for Alcohol and Addiction Studies, Box G, Brown University, Providence, RI 02912. compensatory CRs have been observed to occur in morphine-associated environments in a number of experiments (Hinson \& Siegel, 1983; Paletta \& Wagner, 1986; Siegel, 1983), although they also have failed to occur in situations in which they otherwise would have been expected (Fanselow \& German, 1982; La Hoste, Olson, Olson, \& Kastin, 1980; Tiffany, Petrie, Baker, \& Dahl, 1983).

Most tests of the Pavlovian model of tolerance have measured analgesic or pyretic responses in rats (e.g., Siegel, 1975, 1976, 1977, 1978; Siegel, Hinson, \& Krank, 1978). Typically, a single, brief assessment of morphine-induced analgesia or hyperthermia is made. Since morphine has a variety of effects, some of which endure for hours and some of which are species-specific (Jaffe \& Martin, 1975; Mansky, 1978), it would seem desirable to test the Pavlovian model in other species and response systems. Several studies have been reported in which the Pavlovian model of morphine tolerance has been evaluated in terms of effects on locomotor activity in rats (Fanselow \& German, 1982; Hinson \& Siegel, 1983; Mucha et al., 1981; Paletta \& Wagner, 1986). In the present experiment, we investigated the development of tolerance to morphine's effects on wheel-running activity in the golden Syrian hamster, Mesocricetus auratus.

The effects of morphine on hamster wheel-running activity are well documented: Low doses of morphine produce an increase in activity, whereas high doses produce a decrease. Across a wide range of intermediate doses, morphine elicits first a decrease and then an increase in activity (Schnur, 1985a; Schnur, Bravo, Trujillo, \& Rocha, 1983). With repeated morphine administration, the initial hypoactivity decreases (i.e., tolerance), but the subsequent hyperactivity occurs earlier and increases in magnitude (i.e., reverse tolerance or sensitization) (Schnur, 1985b; Schnur, Bravo, \& Trujillo, 1983). The first purpose in the present experiment was to determine 
whether these changes would prove situation-specific, and if so, whether compensatory CRs would be evident. A second purpose was to test whether nonreinforced exposures to the putative CS following tolerance acquisition would lead to an attenuation of morphine tolerancethat is, extinction of tolerance. Thus, morphine-tolerant animals were either injected with saline and placed in the running-wheel environment (extinction condition) or left undisturbed in the home cage (control condition) for 5 days before a final morphine test. The Pavlovian model predicts that tolerance should be retained by animals spending time in the home cage, but lost or attenuated following exinction training.

\section{METHOD}

\section{Subjects}

Twenty-four adult female golden Syrian hamsters (Mesocricetus auratus) obtained from Sasco, Inc. (Omaha, NE) with a mean weight of $102 \mathrm{~g}$ were used. They were housed individually in wire mesh cages, and maintained on a 12:12 h light:dark cycle (lights on at 7 a.m.). Hamsters were given free access to tap water and paper nesting materials throughout the experiment. They received a daily food ration (Purina Rodent Lab Chow) after each experimental session, sufficient to maintain $90 \%$ of their ad-lib weights.

\section{Apparatus and Materials}

The apparatus consisted of 24 activity wheels (Wahmann Co., Model LC-34) housed in two rooms, each dimly illuminated by two 15-W bulbs. Running wheels were fitted with microswitches and connected via interfaces (Lafayette, Model 1180) to Apple II+ computers to record the number of wheel revolutions. An ambient noise level of $70 \mathrm{~dB}$ (re: 0.0002 dynes $/ \mathrm{cm}^{2}$, A scale) was maintained.

Morphine injections consisted of 50-mg salt $/ \mathrm{kg}$ doses of morphine sulfate and saline injections consisted of a $0.9 \%$ solution of sodium chloride. All injections were administered i.p. in $1-\mathrm{ml} / \mathrm{kg}$ volumes.

\section{Procedure}

The experiment was conducted on 28 consecutive days. During the tolerance development phase of the experiment, hamsters were randomly assigned to three groups $(n=8)$ : Group MRW/SHC received injections of morphine $(50 \mathrm{mg} / \mathrm{kg})$ paired with exposure (40 $\mathrm{min}$ ) to the running-wheel environment and injections of saline in the home cage environment on an alternating day schedule; Group SRW/MHC received injections of saline paired with exposure to the running-wheel environment and injections of morphine in the home cage environment; Group S/S received injections of saline in both environments. The injection procedure during all phases of the experiment was as follows: The animals were confined to the side cage of the Wahmann running wheel and given an injection of either saline or morphine. Ten minutes later, the side cage door was opened, and the animals entered the running wheel. The side cage door was then closed, and the animals were restricted to the running wheel for the duration of the session.

The tolerance development phase lasted 12 days (i.e., 6 morphine and 6 saline injections). On the day following the last cycle, the animals were challenged with a test dose of $50-\mathrm{mg} / \mathrm{kg}$ morphine (Morphine Test 1) in the running-wheel environment. Two days later, the animals were challenged with a test dose of saline (Saline Test) in the running-wheel environment. All test sessions were $3 \mathrm{~h}$ in duration. Following a 3-day rest period, during which all the animals remained undisturbed in the home cages, another morphine test (Morphine Test 2), identical to the first, was conducted.

Two days later, each of the three groups was divided randomly into two subgroups: EXT and HC. Thus, six groups $(n=4)$ were created: Groups MRW/SHC/EXT and MRW/SHC/HC; Groups
SRW/MHC/EXT and SRW/MHC/HC, Groups S/S/EXT and $\mathrm{S} / \mathrm{S} / \mathrm{HC}$. On 5 successive days, the anımals in the EXT subgroups received injections of saline before being placed in the running wheels for a 40-min extinction session. The animals in the HC subgroups remained undisturbed in their home cages during these days. On the day following the last extinction day, a final morphine test (Morphine Test 3), identical to the previous tests, was conducted. The number of wheel revolutions every $20 \mathrm{~min}$ was recorded. All statistical tests were conducted at the $p<.05$ level of significance.

\section{RESULTS}

The top panel of Figure 1 shows mean activity for all groups during Morphine Test 1 . It is evident that, compared with Group $S / S$, animals with prior morphine experience were hyperactive. Group MRW/SHC was hyperactive compared to Groups S/S and SRW/MHC, an indication that morphine tolerance was situation-specific. In addition, Group SRW/MHC was hyperactive compared to Group $\mathrm{S} / \mathrm{S}$, an indication of nonassociative or pharmacological tolerance. A $3 \times 9$ (groups $\times$ time blocks) mixed factorial ANOVA indicated that on Morphine Test 1 , the effect of groups $[F(2,21)=4.76]$ and the effect of time blocks $[F(8,168)=31.54]$ were significant. Subsequent $t$ tests indicated that during the first hour, Group MRW/SHC was more active than Group S/S during Time Blocks 1, 2, and 3 and more active than Group SRW/MHC during Time Block 2. In addition, Group SRW/MHC was more active than Group S/S during Time Blocks 2 and 3. During the second hour, Group MRW/SHC was more active than Group S/S during Time Blocks 4 and 5, and more active than Group SRW/MHC during Time Block 5. Group SRW/MHC was more active than Group S/S during Time Block 4. There were no significant differences among groups during the last hour of the test session.

The middle panel of Figure 1 shows mean activity for all groups during the saline test. It is evident that differences between Group MRW/SHC and Group S/S were small and variable throughout most of the test session. A $3 \times 9$ (groups $\times$ time blocks) mixed factorial ANOVA indicated that during the saline test, the effect of time blocks $[F(8,168)=11.21]$ was significant, but that neither the effect of groups nor the interaction was significant. Thus, the saline test provided little consistent evidence of compensatory CRs.

The bottom panel of Figure 1 shows mean activity for all groups during Morphine Test 2. Associative tolerance is evident in the comparison of Group MRW/SHC with Groups SRW/MHC and S/S. A $3 \times 9$ (groups $\times$ time blocks) mixed factorial ANOVA indicated that the effect of groups $[F(2,21)=7.72]$, the effect of time blocks $[F(8,168)=32.62]$, and the interaction between groups and time blocks $[F(16,168)=2.01]$ were all significant. Subsequent $t$ tests indicated that, during the first hour, Group MRW/SHC was more active than Groups SRW/MHC and S/S during the third time block. During the second hour, Group MRW/SHC was more active than Groups SRW/MHC and S/S during Time Blocks 4, 5, and 6. During the third hour, Group MRW/SHC was more 

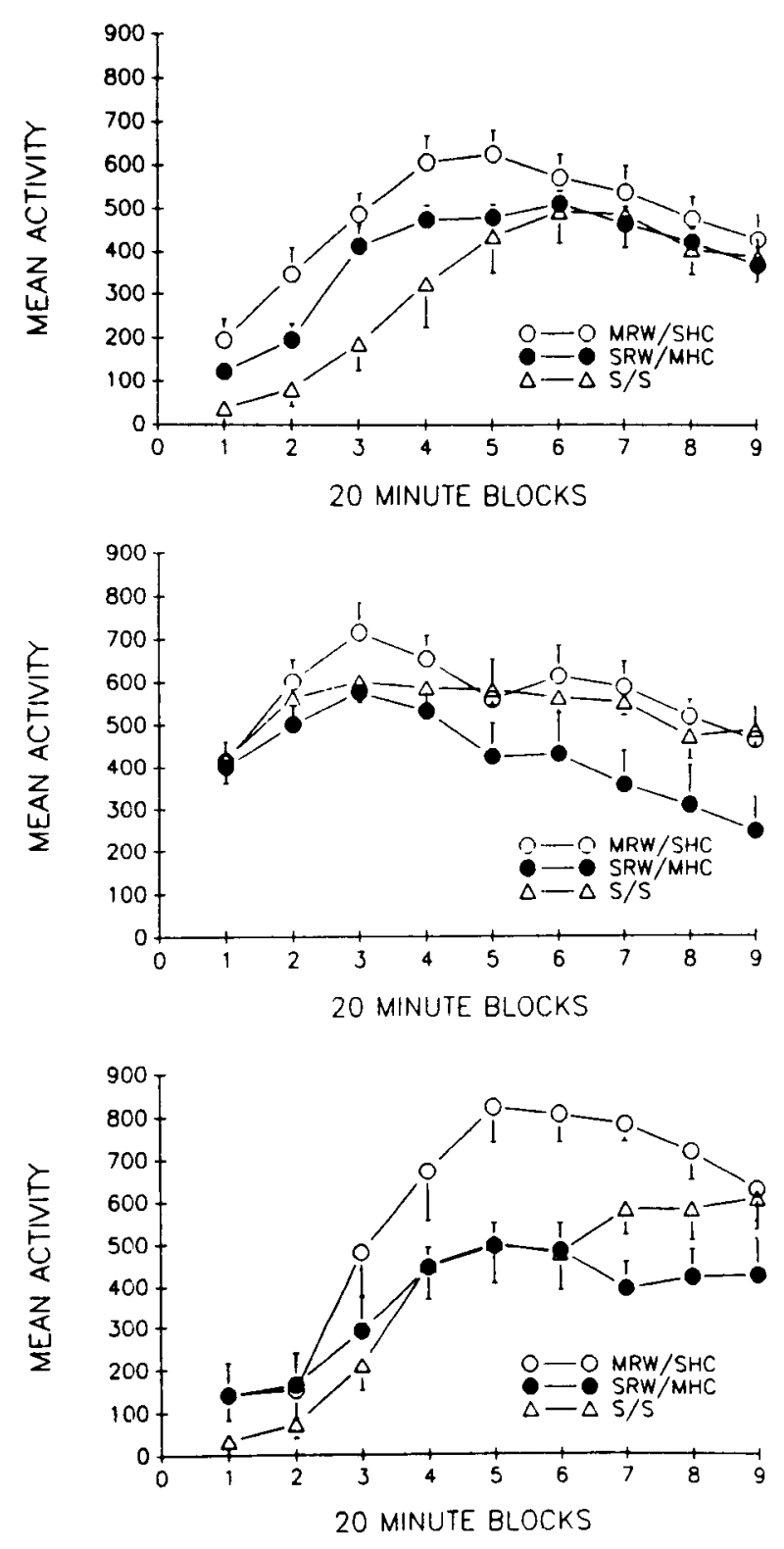

Figure 1. Top panel: Mean activity as a function of 20-min blocks for all groups during Morphine Test 1. Middle panel: Mean activity as a function of 20-min blocks for all groups during the saline test. Bottom panel: Mean activity as a function of 20 -min blocks for all groups during Morphine Test 2. The standard error of the mean is shown for each mean.

active than Group S/S during Time Block 7 , and more active than Group SRW/MHC during Time Blocks 7, 8, and 9. Finally, Group S/S was more active than Group SRW/MHC during Time Block 7.

Figure 2 shows mean activity for all groups during the final morphine test. Each panel depicts the data for one of the three main groups, while the EXT and HC subgroups are plotted separately within the panels. The comparison of most interest concerns Group MRW/SHC, where the predicted effect of extinction training was obtained (see top panel). That is, animals that had 5 days of extinction training (Group MRW/SHC/EXT) were no longer tolerant to morphine-induced hypoactivity, whereas the animals that spent the 5 days undisturbed in the home cage (Group MRW/SHC/HC) retained their morphine tolerance, running at rates similar to those shown during Morphine Test 2 (see Figure 1). For Group SRW/MHC (middle panel) and Group S/S (bottom panel), the extinction treatment had little consistent effect. This was as expected, since neither group had the opportunity to learn an association between cues of the test situation and morphine. Differences between the EXT and HC subgroups therefore were small and variable, though there was some tendency for the EXT subgroups to be more active than their HC counterparts. This might be attributable to a practice effect; the EXT subgroups had a week of daily
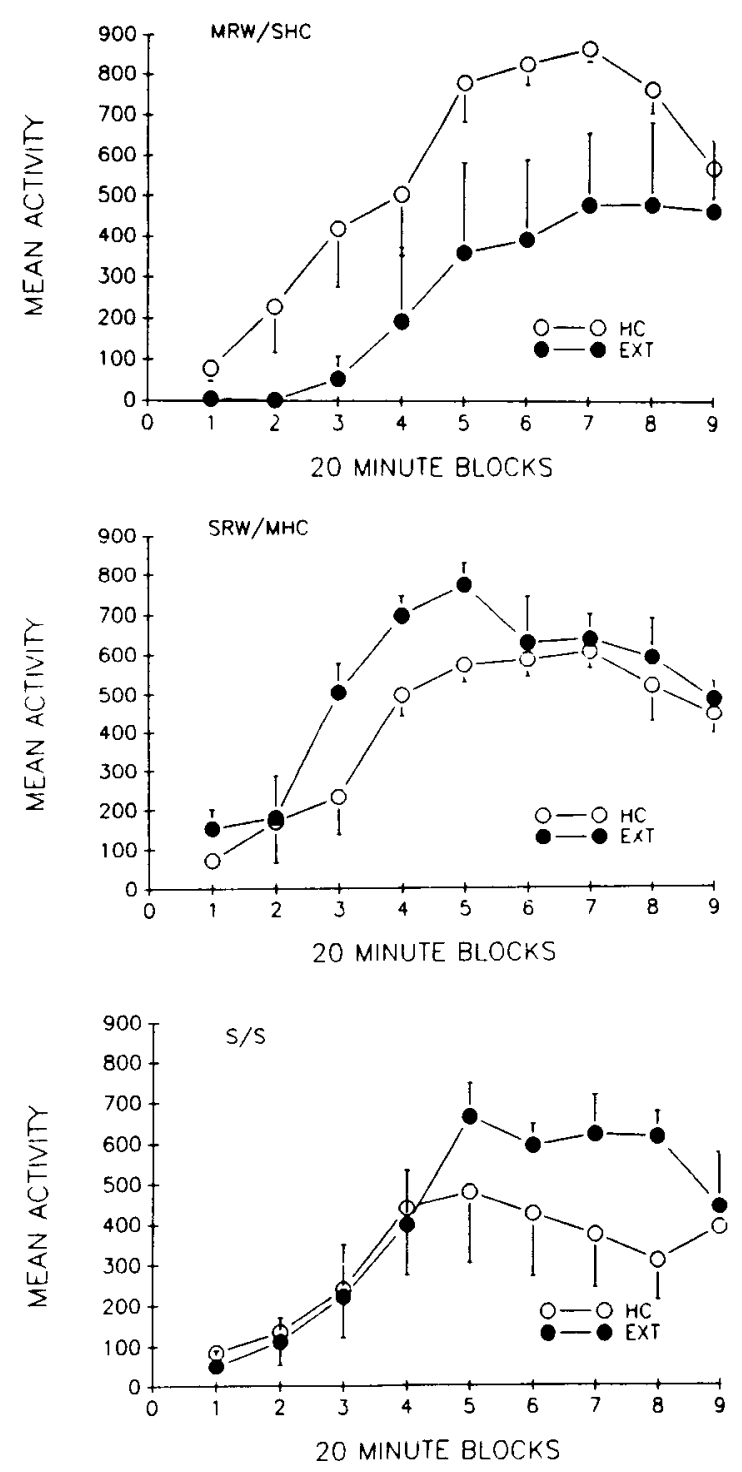

Figure 2. Mean activity as a function of 20-min blocks during the final morphine test. Top panel: Activity for Groups MRW/SHC/EXT and MRW/SHC/HC. Middle panel: Activity for Groups SRW/ MHC/EXT and SRW/MHC/HC. Bottom panel: Activity for Groups S/S/EXT and S/S/HC. The standard error of the mean is shown for each mean. 
running-wheel experience absent in HC subgroups. A $3 \times 2 \times 9$ (groups $\times$ extinction training $\times$ time blocks) mixed factorial ANOVA indicated that the effect of time blocks $[F(8,144)=33.46]$, and the interaction between groups and extinction treatment $[F(2,18)=5.22]$ were significant. Subsequent $t$ tests indicated that, in Group MRW/SHC, the HC subgroup ran at a higher rate than the the EXT subgroup, whereas in Groups SRW/MHC and $S / S$, there were no differences between the EXT and $\mathrm{HC}$ subgroups.

\section{DISCUSSION}

These results, then, provide mixed support for the Pavlovian model of morphine tolerance in the hamster. On the one hand, morphine tolerance was situation-specific, retained for 1 week after the last morphine injection, and attenuated by extinction training. Although Fanselow and German (1982) reported evidence suggestive of the extinction of tolerance to morphine-induced hypoactivity, the failure to employ "home cage" control groups leaves their results inconclusive. In no other study of tolerance to morphine's effects on locomotor activity has the extinction prediction been tested. Thus, the present findings are the first to demonstrate clearly that tolerance to morphine-induced hypoactivity can be attenuated using an extinction procedure.

On the other hand, the present experiment failed to reveal the compensatory CRs postulated by various formulations of the conditioning model of drug tolerance (Kesner \& Baker, 1981; Poulos \& Hinson, 1984; Poulos, Wilkinson, \& Cappell, 1981; Siegel, 1979, 1983). It is well known that compensatory CRs are not always observed concomitantly with situation-specific morphine tolerance (Fanselow \& German, 1982; La Hoste et al., 1980; Tiffany et al., 1983). In studies of locomotor activity in rats, for example, Fanselow and German (1982) were unable to observe compensatory CRs, whereas both Hinson and Siegel (1983) and Paletta and Wagner (1986) did observe them. Mucha et al. (1981) observed compensatory CRs at high doses of morphine, but not at low doses. Thus, there has been considerable disagreement about the conditions required for observing compensatory CRs (Eikelboom \& Stewart, 1982; Mucha et al., 1981; Rochford \& Stewart, 1987).

A number of explanations have been advanced to account for the repeated failures to observe compensatory CRs in situations where they otherwise would be expected. Siegel and MacRae (1984) have proposed that compensatory CRs may not be observable in the absence of the drug itself (i.e., during saline tests), due to self-correcting response mechanisms that mask the compensatory $\mathrm{CR}$. Compensatory responses, however, may be observed in tests using a "priming" drug. For example, Hinson and Siegel (1983) observed compensatory hyperexcitability in the 10-min period following an injection of morphine. Similarly, Hinson, Poulos, and Cappell (1982) inferred the presence of compensatory CRs to pentobarbital sedation during tests in which augmented responses to a prim- ing injection of cocaine were observed. Poulos and Hinson (1984) used a phenobarbital prime to reveal compensatory CRs following the acquisition of tolerance to scopolamineinduced adipsia.

Another explanation for the failure to observe compensatory CRs during saline tests is the suggestion that drug onset cues might be a necessary component of the CS that elicits the CR (Walter \& Riccio, 1983). In the absence of such cues, the compensatory response is not manifested. Thus, saline tests, for one reason or another, might be a relatively insensitive index of compensatory CRs, and our failure to observe them in the present experiment would not be fatal to Pavlovian models (Siegel \& MacRae, 1984).

The difficulty with trying to dismiss failures to observe compensatory CRs by appealing to the insensitivity of saline tests is that such tests have revealed compensatory CRs in other experiments. For example, Siegel (1975, 1977, 1978) obtained evidence for compensatory thermic and analgesic responses during saline tests. In addition, Paletta and Wagner (1986) observed compensatory activity CRs during saline tests. Thus, saline tests are not inherently insensitive, though the conditions necessary for observing compensatory CRs remain obscure (cf. King, Bouton, \& Musty, 1987).

Another class of explanations for the failure to observe compensatory CRs holds that demonstrations of compensatory CRs depend on the response system under investigation (Paletta \& Wagner, 1986; Solomon \& Corbit, 1974) and on how the response system is measured (Krank, 1987). Using a within-groups design, Krank (1987) demonstrated that, in the same animals, compensatory hyperalgesic CRs were observable using paw-lick but not tail-flick indices of pain. Paletta and Wagner (1986) proposed that only response systems with a biphasic response profile to morphine would manifest compensatory CRs (cf. Solomon \& Corbit, 1974). Supporting evidence for this hypothesis was found in their observations of compensatory activity CRs (a biphasic response), but not compensatory analgesic (paw-lick) CRs (a monophasic response). Although the Paletta and Wagner (1986) hypothesis has the virtue of not being a post hoc formulation, it has difficulty accounting for the presence of compensatory CRs in experiments measuring analgesia (Siegel, 1975, 1976) and for the absence of compensatory $\mathrm{CRs}$ in experiments, including the present one, measuring activity (Fanselow \& German, 1982). The failure to observe compensatory CRs in the present experiment also might be explained in terms of a ceiling effect. In contrast to the Paletta and Wagner (1986) study, in which very low rates of responding among control animals were reported, the high levels of activity among saline controls in the present experiment might have precluded the conditioned hyperactivity predicted by the compensatory response hypothesis.

Although Pavlovian models of drug tolerance (Kesner \& Baker, 1981; Poulos \& Hinson, 1984; Poulos et al., 1981; Siegel, 1979, 1983) specifically predict the elicitation of compensatory CRs by drug-associated cues, the 
observation of such responses would not provide unequivocal support for the model, even if such observations were consistently reported. This is clear particularly for biphasic responses such as locomotor activity. As we have found in the hamster (Schnur et al., 1983), and as others have found in the rat (Babbini \& Davis, 1972; Paletta \& Wagner, 1986), morphine elicits both hypoactivity and hyperactivity, depending on dose and time since administration. Moreover, these two responses seem to be mediated separately by different brain sites (Joyce \& Iversen, 1979). Since hyperactivity can occur independently of hypoactivity, it is evident that morphine-elicited hyperactivity is not necessarily compensatory for morphineelicited hypoactivity. Furthermore, Vezina and Stewart (1984) have demonstrated that hyperactivity elicited by morphine administration in the ventral tegmentum can be conditioned to environmental cues. Thus, the observation that morphine-associated cues elicit hyperactivity following saline (or drug for that matter) might reflect the direct conditioning of hyperactivity rather than the conditioned elicitation of compensatory responses (cf. Tabakoff \& Kiianmaa, 1982).

The considerations above indicate that empirical documentation of compensatory responses is neither necessary nor sufficient for confirmation/refutation of the Pavlovian conditioning model. It is not necessary, because there are mitigating factors that can be invoked to account for failures to observe the predicted CRs (Siegel \& MacRae, 1984; Walter \& Riccio, 1983). It is not sufficient, since, as in the case of biphasic responses, it is impossible to prove that the observed responses are indeed compensatory. Thus, the Pavlovian model of morphine tolerance is weakened, not by the evidence, but by the inability of the evidence to decisively confirm or refute the compensatory response postulate. The same weakness is shared by other models that incorporate a compensatory response postulate (Kesner \& Baker, 1981; Poulos \& Hinson, 1984).

An alternative approach is to abandon the compensatory response postulate, while retaining the associative framework of the conditioning model. For example, Baker and Tiffany (1985) have proposed a habituation model of morphine tolerance, which makes many of the same predictions as the Pavlovian model in most situations (Tiffany \& Maude-Griffin, 1988), but which is neutral with regard to the directionality of CRs. The habituation model of morphine tolerance, which is derived from Wagner's (1979) priming model of habituation, proposes that tolerance reflects learned and unlearned changes in the processing of drug-produced stimuli. In addition to avoiding the compensatory response problem, the model has the virtue of explaining both associative and nonassociative tolerance. Since associative and nonassociative tolerance often occur concomitantly, as they did in the present experiment, a model attempting to integrate both phenomena in a single framework would seem parsimonious. Recent evaluations of the habituation model (Tiffany \& Maude-Griffin, 1988) have been encouraging. It re- mains for future research to determine whether the habituation model can account for a wide range of phenomena of associative and nonassociative tolerance.

\section{REFERENCES}

Babbini, M., \& Davis, W. M. (1972). Time-dose relations for locomotor effects of morphine after acute or repeated treatment. British Journal of Pharmacology, 46, 213-224.

Baker, T. B., \& Tiffany, S. T. (1985). Morphine tolerance as habituation. Psychologıcal Review, 92, 78-108.

Eikelboom, R., \& STEWART, J. (1982) The conditioning of druginduced physiological responses. Psychological Review, 89, 507-528.

Fanselow, M. S., \& German, C. (1982). Explicitly unpaired delivery of morphine and the test situation: Extinction and retardation of the suppressing effects of morphine on locomotor activity. Behavioral \& Neural Science, 35, 231-242.

Goudie, A J., \& DemellweEk, C. (1986). Conditioning factors in drug tolerance. In S. R. Goldberg \& I. P. Stolerman (Eds.), Behavioral analysis of drug dependence (pp. 225-285). New York: Academic Press.

Hinson, R. E., Poulos, C. X , \& Cappell, H. (1982). Effects of pentobarbital and cocaine in rats expecting pentobarbital. Pharmacology, Blochemistry \& Behavior, 16, 661-666.

Hinson, R. E., \& SiEgel, S. (1983). Anticipatory hyperexcitability and tolerance to the narcotizing effects of morphine in the rat. Behavioral Neuroscience, 97, 759-767.

JAFFE, J. H., \& MARTIN, R. (1975). Narcotic analgesics and antagonists. In L. S. Goodman \& A. Gilman (Eds.), The pharmacological basis of therapeutics (5th ed., pp. 248-283). New York: Macmillan.

JoYCE, E. M., IVERSEN, S. D. (1979). The effect of morphine applied locally to mesencephalic dopamine cell bodies on spontaneous motor activity in the rat. Neuroscience Letters, 14, 207-212.

KeSNER, R. P., \& BAKER, T. B. (1981). A two-process model of tolerance. In J. L. Martinez, R. A. Jensen, R. B. Messing, H. Rigter, \& J. L. McGaugh (Eds.), Endogenous peptides and learning and memory processes (pp. 479-518). New York: Academic Press.

King, D. A., Bouton, M. E., \& Musty, R. E. (1987). Associative control of tolerance to the sedative effects of a short-acting benzodiazepine. Behavioral Neuroscience, 101, 104-114.

KraNK, M. D. (1987). Conditioned hyperalgesia depends on the pain sensitivity measure. Behavioral Neuroscience, 101, 854-857.

La Hoste, G. J., Olson, R. D., Olson, G. A., \& Kastin, A. J. (1980). Effects of Pavlovian conditioning and MIF-1 on the development of morphine tolerance in rats. Pharmacology, Biochemistry \& Behavior, 13, 799-804.

MaNSKY, P. A. (1978). Human psychopharmacology. In L. L. Iversen, S. D. Iversen, \& S. H. Snyder (Eds.), Handbook of psychopharmacology: Drugs of abuse (Vol. 10, pp. 95-185). New York: Plenum.

Mucha, R. F., Volkovskis, C., \& Kalant, H. (1981). Conditioned increases in locomotor activity produced with morphine as an unconditioned stimulus, and the relation of conditioning to acute morphine effect and tolerance. Journal of Comparative \& Physiological Psychology, 95, 351-362.

PaletTA, M. S., \& Wagner, A. R. (1986). Development of contextspecific tolerance to morphine: Support for a dual-process interpretation. Behavioral Neuroscience, 100, 611-623.

Poulos, C. X., \& Hinson, R. E. (1984). A homeostatic model of Pavlovian conditioning: Tolerance to scopolamine-induced adipsia. Joumal of Experimental Psychology: Animal Behavior Processes, 10, 75-89.

Poulos, C. X., Wilkinson, D. A., \& CAPPEll, H. (1981). Homeostatic regulation and Pavlovian conditioning in tolerance to amphetamineinduced anorexia. Journal of Comparative \& Physiological Psychology, 95, 735-746.

RoCHFord, J., \& STEWART, J. (1987). Morphine attenuation of conditioned autoanalgesia: Implications for theories of situation-specific tolerance to morphine analgesia. Behavioral Neuroscience, 101 , 690-700.

SCHNUR, P. (1985a). Effects of naloxone and naltrexone on morphine 
elicited changes in hamster locomotor activity. Physiological Psychology, 13, 26-32.

SCHNUR, P. (1985b). Morphine tolerance and sensitization in the ham ster. Pharmacology, Biochemistry \& Behavior, 22, 157-158.

Schnur, P., Bravo, F., \& Trujillo, M. (1983). Tolerance and sensitization to the biphasic effects of low doses of morphine in the hamster. Pharmacology, Biochemistry \& Behavior, 19, 435-439.

Schnur, P., Bravo, F., Trujillo, M., \& Rocha, S. (1983). Biphasic effects of morphine on locomotor activity in hamsters. Pharmacology, Biochemistry \& Behavior, 18, 357-361.

SIEGEL, S. (1975). Evidence from rats that morphine tolerance is a learned response. Journal of Comparative \& Physiological Psychology, 89, 498-506.

SIEGEL, S. (1976). Morphine analgesic tolerance: Its situation specificity supports a Pavlovian conditioning model. Science, 193, 323-325.

SIEGEL, S. (1977). Morphine tolerance acquisition as an associative process. Journal of Experimental Psychology: Animal Behavior Processes, 3, 1-13.

SIEGEL, S. (1978). Tolerance to the hyperthermic effect of morphine in the rat is a learned response. Journal of Comparative \& Physiological Psychology, 92, 1137-1149.

SIEGEL, S. (1979). The role of conditioning in drug tolerance and addiction. In J. D. Keehn (Ed.), Psychopathology in animals: Research applications (pp. 143-168). New York: Academic Press.

SIEGEL, S. (1983). Classical conditioning, drug tolerance, and drug dependence. In Y. Israel, F. B. Sloser, H. Kalant, R. E. Popham, W. Schmidt, \& R. G. Smart (Eds.), Research advances in alcohol and drug abuse (Vol. 7, pp. 207-246). New York: Plenum.

Siegel, S., Hinson, R. E., Krank, M. D. (1978). The role of predrug signals in morphine analgesic tolerance: Support for a Pavlovian conditioning model of tolerance. Journal of Experimental Psychology: Animal Behavior Processes, 4, 188-196.

SiEgeL, S., \& MACRAE, J. (1984). Environmental specificity of tolerance. Trends in Neuroscience, 7, 140-143.
Solomon, R. L., \& Corbit, J. D. (1974). An opponent process theory of motivation: I. Temporal dynamics of affect. Psychological Review, 81, 119-145.

Stewart, J., Eikelboom, R. (1987). Conditioned drug effects. In L. L. Iversen, S. D. Iversen, \& S. H. Snyder (Eds.), Handbook of psychopharmacology: New directions in behavioral pharmacology (Vol. 19, pp. 1-57). New York: Plenum.

Tabakoff, B., KiianmaA, K. (1982). Does tolerance develop to the activating as well as to the depressant effects of alcohol? Pharmacol ogy, Biochemistry \& Behavior, 17, 1073-1076.

TIFfany, S. T., \& BAKER, T. B. (1981). Morphine tolerance in rats: Congnuence with a Pavlovian paradigm. Journal of Comparative \& Physiological Psychology, 95, 747-762.

Tiffany, S. T., \& Maude-Griffin, P. M. (1988). Tolerance to morphine in the rat: Associative and nonassociative effects. Behavioral Neuroscience, 102, 534-543.

Tiffany, S. T., Petrie, E. C., Baker, T. B., \& Dahl, J. L. (1983) Conditioned morphine tolerance in the rat: Absence of a compensatory response and cross-tolerance with stress. Behavioral Neuroscience, 97, 335-353.

Vezina, P., Stewart, J. (1984). Conditioning and place-specific sensitization of increases in activity induced by morphine in the VTA. Pharmacology, Biochemistry \& Behavior, 20, 925-934.

WAGNER, A. R. (1979). Habituation and memory. In A. Dickinson \& R. A. Boakes (Eds.), Mechanisms of learning and motivation (pp. 5382). Hillsdale, NJ: Erlbaum.

Walter, T. A., \& Riccio, D. C. (1983). Overshadowing effects in the stimulus control of morphine analgesic tolerance. Behavioral Neuroscience, 97, 658-662.

(Manuscript received July 5, 1988;

revision accepted for publication December 13,1988 .) 